\title{
22 Dank
}

Eine Dissertation entsteht im jahrelangen Austausch mit ganz unterschiedlichen Personen aus dem beruflichen und privaten Umfeld. Es sind diese zahlreichen Begegnungen und Anregungen, die Kritik und Zuspruch bringen und über die Zeit hinweg nicht nur den Entwicklungsprozess eines Dissertationsprojekts, sondern auch den der Verfasserin maßgeblich beeinflussen. All jenen Menschen, die mich im Verlauf des Dissertationsprojekts unterstützt haben, möchte ich ganz herzlich danken. Es sind zu viele, um alle einzeln nennen zu können.

Als Erstgutachterin war Marianne Sommer in allen Phasen des Dissertationsprojekts meine erste Ansprechperson. Dank ihres geduldigen Rückhalts wurde ich von ihr bei meinen Entscheidungen stets unterstützt. Besonders schätzte ich ihr Vertrauen, das sie mir in unseren konstruktiven Gesprächen entgegenbrachte, sodass ich die Dissertation entsprechend meinen Vorstellungen und Ideen entwickeln durfte.

Meiner Zweitgutachterin, Beate Hochholdinger-Reiterer, möchte ich für ihr großes Interesse an meinem Forschungsvorhaben und ihr unablässiges Engagement für mein Projekt danken. Sie hat mich von Beginn an unterstützt und mich mit hilfreichen Bemerkungen und wertvollen Hinweisen gefördert.

Beiden Betreuerinnen möchte ich einen besonderen Dank aussprechen. Mit ihren kritischen Anregungen haben sie mich gelehrt, die eigenen Erkenntnisse immer wieder aufs Neue zu hinterfragen und weiterzuentwickeln.

Bei der Graduate School of Humanities and Social Science der Universität Luzern sowie bei Herrn Kurt W. Meyer, Stiftungsratsmitglied des Luzerner Theaters, bedanke ich mich für die Anschubfinanzierung in der ersten Entwicklungsphase meines Dissertationsprojekts.

Dem Schweizerischen Nationalfonds danke ich für die Teilfinanzierung des Forschungsaufenthalts am Max Planck-Institut für Bildungsforschung (MPIB) im Forschungsbereich Geschichte der Gefühle in Berlin und für die Finanzierung der Publikation.

Ute Frevert leitet am MPIB einen außergewöhnlichen Forschungsbereich. Im internationalen Austausch konnte ich dort meine Forschung in einem intellektuell höchst anregenden Umfeld betreiben, mich im kollegialen Austausch fortbilden und weiterentwickeln. Ute Frevert hat mir neue Perspektiven auf meinen Forschungsgegenstand eröffnet und mir geholfen, meine Argumentation präzis zu fassen. Wichtige Ansprechpersonen am MPIB zur kritischen Auseinandersetzung und Weiterentwicklung der Forschungsfragen waren Edgar Cabanas, Juliane Brauer, Monika Freier, Benno Gammerl, Bettina Hitzer und Anja Laukötter. Bei allen möchte ich mich herzlich für ihr außergewöhnliches Interesse an meinem

Ә OpenAccess. (c) 2021 Isabelle Haffter, publiziert von De Gruyter. (cc) BY-NC-ND Dieses Werk ist lizenziert unter einer Creative Commons Namensnennung - Nicht kommerziell - Keine Bearbeitung 4.0 International Lizenz. https://doi.org/10.1515/9783110661439-022 
Projekt bedanken. Für die vielen Gespräche und die große Herzlichkeit möchte ich den Doktorand*innen am MPIB, insbesondere Sören Brandes, Alexandra Esche, Thomas Linder, Marvin Bähr und Sander Tordoir danken.

Bei allen Leiter*innen und Teilnehmer*innen der verschiedenen Tagungen, Workshops und Kolloquien, mit denen ich über mein Projekt diskutieren durfte, bedanke ich mich für die wertvollen Anregungen und Hinweise.

Ebenfalls möchte ich mich bei allen Archivar^innen und Bibliothekar^innen bedanken, die mir den Zugang zu dem wertvollen Quellenmaterial gewährt und mich bei meinen Recherchefragen mit ihrem unschätzbaren Wissen unterstützt haben.

Meinen Freund ${ }^{\star}$ innen, die mich über die Jahre hinweg bei meinem Vorhaben moralisch unterstützt haben, bedanke ich mich herzlich.

Meine Eltern Adèle und Damian Haffter und mein Bruder Christoph Haffter waren stets verständnisvolle Begleitende und geduldige Unterstützende meines Vorhabens und sind mir mit Rat und Tat zur Seite gestanden. Ihnen gilt mein besonderer Dank.

Matthias Jakobs, mein Lebens- und Gesprächspartner, hat mich bei meinem Vorhaben unablässlich unterstützt. Seine Liebe und sein Zuspruch haben mich darin bestärkt, das Forschungsprojekt durchzuführen, wie es jetzt als Manuskript vorliegt.

Für die Realisierung des Buchprojekts und die redaktionelle Betreuung danke ich dem De Gruyter Verlag. Ein großer Dank für die gute Zusammenarbeit geht an Julia Brauch und Jana Fritsche.

Basel, 22. Januar 2021 